\title{
EXACT SEQUENCES FOR GENERALIZED TOEPLITZ OPERATORS
}

\author{
CARL SUNDBERG
}

(Communicated by John B. Conway)

\begin{abstract}
Let $\tau$ be the $C^{*}$-algebra generated by the Toeplitz operators on $H^{2}$ of the unit circle, and let $C$ be the $\tau$-ideal generated by $\left\{T_{\varphi} T_{\psi}-\right.$ $\left.T_{\varphi \psi}: \varphi, \psi \in L^{\infty}\right\}$. It is well known that $\tau / C$ is naturally *-isomorphic to $L^{\infty}$. Several authors have obtained a similar result for other classes of Toeplitz operators. In the present paper a general theorem is proved which establishes the relevant isomorphism for a wide class of generalized Toeplitz operators.
\end{abstract}

1. Introduction. In the study of Toeplitz operators $T_{\varphi}$ on the Hardy space $H^{2}$ on the unit circle, two objects of interest are the Toeplitz algebra $\tau$, which is the $C^{*}$-operator algebra generated by the Toeplitz operators, and its semicommutator ideal $C$, which is the $\tau$-ideal generated by $\left\{T_{\varphi} T_{\psi}-T_{\varphi \psi}: \varphi, \psi \in L^{\infty}\right\}$. A basic fact concerning these objects is that $\tau / C$ is naturally isomorphic to $L^{\infty}$, and a related fact is the spectral inclusion theorem: $R(\varphi) \subset \sigma\left(T_{\varphi}\right)$, where $R(\varphi)$ denotes the essential range of $\varphi$. For proofs of these facts and some consequences, see Chapter 7 of $[3]$.

Recently there has been interest in the study of operators similar to the classical Toeplitz operators which act on Hilbert spaces of analytic functions other than $H^{2}$. For many of these operators the isomorphism $\tau / C$ with some naturally related function algebra is proven and a related spectral inclusion theorem is shown; see for example [1, 2, and 6]. It is the purpose of this note to show how an idea of Davie and Jewell in [2] can be used to prove an isomorphism theorem for a very general class of operators. This result in fact shows that in all cases, the isomorphism theorem and the spectral inclusion theorem are equivalent.

2. The main result. Let $\mu$ be a positive measure on some measure space, $H$ a closed subspace of $L^{2}(\mu)$, and $P$ the orthogonal projection of $L^{2}(\mu)$ onto $H$. To $\varphi \in L^{\infty}(\mu)$ we associate the generalized Toeplitz operator $T_{\varphi}: H \rightarrow H$ defined by

$$
T_{\varphi} f=P(\varphi f) \quad \text { for } f \in H .
$$

Thus $T_{\varphi}=P M_{\varphi} \mid H$, where $M_{\varphi}$ is the operation of multiplication by $\varphi$.

Now let $A$ be a $C^{*}$-subalgebra of $L^{\infty}(\mu)$, with maximal space $\mathcal{M}$. For notational convenience, if $\varphi \in A$ and $x \in \mathcal{M}$ we will write $\varphi(x)$ for the action of $x$ on $\varphi$. Let $\tau$ be the $C^{*}$-operator algebra generated by $\left\{T_{\varphi}: \varphi \in A\right\}$, and let $C$ be the $\tau$-ideal

Received by the editors September 2, 1986.

1980 Mathematics Subject Classification (1985 Revision). Primary 47B35, 47D25.

Key words and phrases. Toeplitz operators, semicommutator ideal.

The author is partially supported by NSF Grant \#DMS 8401913. 
generated by $\left\{T_{\varphi} T_{\psi}-T_{\varphi \psi}: \varphi, \psi \in A\right\}$. We then have

THEOREM. $T / C$ is naturally $*$-isometrically isomorphic to $C\left(\mathcal{M}_{1}\right)$, where

$$
\mathcal{M}_{1}=\left\{x \in \mathcal{M} \mid \varphi \in A, \varphi(x)=0 \Rightarrow M_{\varphi} \text { is not bounded below on } H\right\} .
$$

That is, the sequence

$$
0 \rightarrow I \rightarrow A \stackrel{\Phi}{\rightarrow} \tau / \mathcal{C} \rightarrow 0
$$

is exact, where $\Phi(\varphi)=T_{\varphi}+C$ and $I=\left\{\varphi \in A \mid \varphi \equiv 0\right.$ on $\left.\mathcal{M}_{1}\right\}$.

Proof. We first observe that $\varphi \mapsto T_{\varphi}$ is linear and satisfies

(a) $T_{1}=I$,

(b) $\left\|T_{\varphi}\right\| \leq\left\|M_{\varphi} \mid H\right\| \leq\|\varphi\|_{\infty}$,

(c) $T_{\varphi}^{*}=T_{\bar{\varphi}}$,

(d) $\left\|T_{\varphi} f\right\|_{2}^{2} \leq\left\|M_{\varphi} f\right\|^{2} \leq\|\varphi\|_{\infty}\left\langle T_{|\varphi|} f \mid f\right\rangle$ for $f \in H$,

(e) $\varphi \geq 0 \Rightarrow T_{\varphi} \geq 0$.

Only the second inequality in (d) requires any comment: if $f \in H$, then

$$
\begin{aligned}
\left\|M_{\varphi} f\right\|_{2}^{2} & =\int|\varphi f|^{2} d \mu \leq\|\varphi\|_{\infty} \int|\varphi||f|^{2} d \mu \\
& =\|\varphi\|_{\infty}\langle|\varphi| f \mid f\rangle=\|\varphi\|_{\infty}\left\langle T_{|\varphi|} f \mid f\right\rangle .
\end{aligned}
$$

It is an easy consequence of (b) that $\mathcal{M}_{1}$ is closed. By the definition of $C, \Phi$ is an algebra homomorphism. Define $\mathcal{M}_{2}$ to be the zero set of the ideal $\operatorname{ker} \Phi \subset A$. The content of the Theorem is that $\mathcal{M}_{2}=\mathcal{M}_{1}$.

To show that $\mathcal{M}_{1} \subseteq \mathcal{M}_{2}$ we use a direct adaptation of the proof of Theorem 2.2 in [2]. Let $x_{0} \in \mathcal{M}_{1}$ and let $\varphi \in A$ be such that $\varphi\left(x_{0}\right)=1$. We wish to show that $T_{\varphi} \notin C$; to this end, let

$$
S=\sum_{j}\left(\prod_{i} T_{\xi_{j}^{(i)}}\right)\left(T_{\eta_{i}} T_{\psi_{j}}-T_{\eta_{j} \psi_{j}}\right)\left(\prod_{l} T_{k_{j}^{(l)}}\right)
$$

be a typical generating element of $\mathcal{C}$. Relabel the function $\varphi$ as $\varphi_{0}$ and relabel all the functions $\xi_{j}^{(i)}, \eta_{j}, \psi_{j}, \eta_{j} \psi_{j}, k_{j}^{(l)}$ as $\varphi_{1}, \ldots, \varphi_{N}$. Define $\Psi=\sum_{k=0}^{N}\left|\varphi_{j}-\varphi_{j}\left(x_{0}\right)\right|$. Then $\Psi\left(x_{0}\right)=0$, so since $x_{0} \in \mathcal{M}_{1}$ there exist $f_{n} \in H$ such that $\left\|f_{n}\right\|_{2}=1$ and $\left\|T_{\Psi} f_{n}\right\|_{2} \rightarrow 0$. By (e) $T_{\left|\varphi_{j}-\varphi_{j}\left(x_{0}\right)\right|} \geq 0$, hence $\left\|T_{\left|\varphi_{j}-\varphi_{j}\left(x_{0}\right)\right|} f_{n}\right\|_{2} \rightarrow 0$. By (d) and (a) this implies that $\left\|T_{\varphi_{j}} f_{n}-\varphi_{j}\left(x_{0}\right) f_{n}\right\|_{2} \rightarrow 0, j=0, \ldots, N$. This shows that $\left\|S f_{n}\right\|_{2} \rightarrow 0$ and $\left\|T_{\varphi} f_{n}\right\| \rightarrow 1$, so $\|T-S\| \geq 1$. Since $S$ was a typical generating element of $C$, this shows that $T_{\varphi} \notin C$. We have shown that $\varphi\left(x_{0}\right)=1$ implies $T_{\varphi} \notin C$, which shows that $x_{0} \in \mathcal{M}_{2}$.

To show that $\mathcal{M}_{2} \subseteq \mathcal{M}_{1}$, let $x \in \mathcal{M} \backslash \mathcal{M}_{1}$. There then exists a $\varphi \in A,\|\varphi\|_{\infty}=1$, such that $\varphi\left(x_{0}\right)=0$ and $M_{\varphi}$ is bounded below on $H$. By (d), $T_{|\varphi|}$ is then bounded below. This together with (b) and (e) implies that $\left\|I-T_{|\varphi|}\right\|<1$, so $T_{|\varphi|}$ is invertible in $\tau$. By the definition of $\mathcal{M}_{2}$ this implies that $|\varphi|$ does not vanish anywhere on $\mathcal{M}_{2}$. Since $\varphi\left(x_{0}\right)=0$ this shows that $x_{0} \notin \mathcal{M}_{2}$.

REMARK. By (d) and (e), $T_{|\varphi|}$ is invertible iff $M_{|\varphi|}$ is bounded below on $H$. Hence an equivalent definition of $\mathcal{M}_{1}$ is that $\mathcal{M}_{1}$ is the largest of those subsets $E \subset \mathcal{M}$ such that $\varphi(E) \subset \sigma\left(T_{\varphi}\right)$ for all $\varphi \in A$. 
3. Some examples. If $H \subset L^{2}(\mu)$ has the property that whenever $\mu(E)>0$ there exist $f_{n} \in H,\left\|f_{n}\right\|_{2}=1$ such that $\int_{E}\left|f_{n}\right|^{2} d \mu \rightarrow 1$, then the Theorem shows that $\tau / \mathcal{C} \approx A$, since in this case $\mathcal{M}_{1}=\mathcal{M}$. The examples in (i) and (ii) below are of this type; the necessary calculation for (i) is easy and standard, and the calculation for (ii) is done in the proof of Theorem 2.1 of [2].

(i) Take $\mu=d \theta$ on $[|z|=1], H=H^{2}$. Then $\tau / C \approx A$. The standard cases are $A=L^{\infty}$ and $A=C$, the continuous functions on $[|z|=1]$. See Chapter 7 of [3].

(ii) Take $\mu$ to be surface measure on $S$, the boundary of the unit ball in $\mathbf{C}^{n}$, and $H=H^{2}(S)$. Then $\tau / C \approx A$. The case $A=L^{\infty}$ was done by Davie and Jewell [2] and the case $A=C(S)$ was done by Coburn [1].

(iii) Take $\mu$ to be two-dimensional Lebesgue measure on $[|z|<1]$ and let $H$ be the Bergman space of analytic $L^{2}(\mu)$ functions. We consider two possibilities for the algebra $A$.

$A=L^{\infty}$ : We consider $\mathcal{M}$ to be the space of ultrafilters on the lattice of measurable sets modulo sets of measure zero. Then $\tau / C \approx C\left(\mathcal{M}_{1}\right)$ where $\mathcal{M}_{1}$ consists of those ultrafilters $\mathcal{E}$ having the property that if $E \in \mathcal{E}$ and $\varepsilon>0$ there exists a "Carleson square" $S_{h}=\left\{z=r e^{i \theta}|1-h<r<1,| \theta-\theta_{0} \mid<h\right\}$ such that $\mu\left(E \cap S_{h}\right)>(1-\varepsilon) S_{h}$. The necessary calculations follow from a theorem of Hastings [4]; see also [6].

$A$ equals the $C^{*}$-algebra generated by $H^{\infty}$ : In this case $\mathcal{M}$ is the maximal ideal space of $H^{\infty}$ and $\mathcal{M}_{1}$ is the set of "one-point parts" of $\mathcal{M}$ (see [5]). This fact was established in [6]; the use of the present Theorem allows for a considerable simplification of the proof.

\section{REFERENCES}

1. L. A. Coburn, Singular integral operators and Toeplitz operators on odd spheres, Indiana Univ. Math. J. 23 (1973), 433-439.

2. A. M. Davie and N. P. Jewell, Toeplitz operators in several complex variables, J. Funct. Anal. 26 (1977), 356-368.

3. R. G. Douglas, Banach algebra techniques in operator theory, Academic Press, New York, 1972.

4. W. W. Hastings, A Carleson measure theorem for Bergman spaces, Proc. Amer. Math. Soc. 52 (1975), 237-241.

5. K. Hoffman, Bounded analytic functions and Gleason parts, Ann. of Math. (2) 86 (1967), 74-111.

6. G. McDonald and C. Sundberg, Toeplitz operators on the disc, Indiana Univ. Math. J. 28 (1979), 595-611.

Department of Mathematics, University of Tennessee, Knoxville, TenNESSEE 37916 